\title{
Time of Outage Restoration Analysis in Distribution Systems
}

\author{
Mo-yuen Chow \\ IEEE Senior Member \\ Dept. of Elect. and Comp. Engr. \\ North Carolina State University \\ Raleigh, NC 27695
}

\author{
Leroy S. Taylor \\ IEEE Member \\ Distribution Standards \\ Duke Power Company \\ Charlotte, NC 28201-1010
}

\author{
Mo-Suk Chow \\ Non-Member \\ Department of MSIS \\ Penn State University \\ University Park, PA 16802
}

\begin{abstract}
This paper presents an approach that uses statistical techniques to analyze and interpret Time of Outage Restoration (TOR) data in distribution systems with respect to several selected factors. The factors are classified into three main categories : (1) Time, (2) Consequence, and (3) Environment. Each category is further broken down into groups and classes for more detailed analysis. Both statistical, quantitative results and field engineering insights will be correlated and discussed. The approach taken in this paper can be easily modified for other utilities' TOR analysis. These analyses can provide a better understanding as to how the restoration time is affected by different influential factors. The methodology and general discussion in the paper will be useful for future research and development of the Time of Outage Restoration issues in distribution systems, such as model development for customer service improvement, distribution operations, and planning.
\end{abstract}

Key Words : distribution systems, outages, restoration time, statistical analyses.

\section{Introduction}

One of the primary missions of power companies is to provide a reliable, economical and safe supply of electricity to the customers. With the growing usage of electricity by the public, the complexity of power system operations is increasing at a fast rate. In order to achieve its mission with a high standard, power systems nowadays rely heavily on the refinement of conventional models, techniques and experience, extensive usage of computers, and the application of hightech, emerging technologies, along with research in various other areas, in order to meet its current and future challenges $[1,2]$.

Power distribution system operations require a major amount of man power and resources to properly maintain the systems' daily functions. These operations are highly unstructured, nonlinear, stochastic and time varying (compared to generation systems and transmission systems). In addition, distribution systems cover larger physical areas, thus increasing the chances and the number of unexpected events that can occur to these systems. The large exposure of the distribution systems to outdoor environments makes them difficult to analyze, model and, at the same time, more vulnerable to natural disturbances.

Distribution faults have a large impact on power systems' reliability, security and quality, among other important factors.

96 WM 081-0 PWRD A paper recommended and approved by the IEEE Transmission and Distribution Committee of the IEEE Power Engineering Society for presentation at the 1996 IEEE/PES Winter Meeting, January 21 25, 1996, Baltimore, MD. Manuscript submitted July 14, 1995; made available for printing November $30,1995$.
Most utility companies experience a large number of distribution faults in their systems. Customers (industrial, commercial, and residential) are very concerned about the duration of the outages because outages can directly affect their economic revenue, production rate, safety, and convenience. Therefore, when an outage occurs, one of the most common questions that customers may ask is : How long will it take to restore the power? We term this as the Time of Outage Restoration (TOR) problem in this article. Currently, when an outage occurs and customers call in to report the outage, they usually request for an estimate of the TOR. In general, operators will make an educated guess about the restoration time based on their previous experiences and other available information. This approach does not objectively and efficiently utilize available information and data, and may lead to a large error variance between the estimate and the actual outage duration.

If we are able to provide a reasonable estimate of the TOR, then customers will be much happier because they can make contingency plans to minimize the negative effects of the outage on their side. With a better understanding of the effects of different influential factors on TOR, we certainly can provide a better estimate of the TOR. However, to provide an objective and accurate TOR estimate is a challenging task because it depends on so many different factors, such as weather conditions, time of occurrence, the fault causes, the availability of manpower, the number of outages need to be restored at that time, the location of the outage, etc. To further complicate the TOR analysis, different influential factors may have direct and indirect effects on the restoration time. For example, a stormy weather may cause more lightning strike faults. It is very difficult to isolate only direct influential factors for TOR analysis. It is unrealistic for this paper to investigate and report all possible factors that can influence TOR. However, we can develop and present a general approach to correctly extract factors that influence TOR for further analysis, so that the TOR estimate can be improved.

In addition to providing better customer services, the utility can also be benefited with better understanding of Time of Outage Restoration under different scenarios. For example, the crew assignment and outage restoration process can be scheduled more efficiently and effectively, thus increasing the reliability of the distribution systems and reducing the operation costs.

Some utility companies have been collecting data related with outage restoration time. Outage data is recorded on the Duke Power Distribution System each time that an outage occurs as a result of the operation of a breaker relay, fuse, or another protective device. The recorded data contains information such as the geographical location, circuit ID number, weather condition, time of the fault occurrence, and the time of power restoration. Twenty-two information items 
are recorded for each outage at Duke Power Company. Some of the information is based on a coding system, designed by Duke Power engineers and summarized in charts, that describe the circumstances under which the outages occurred (e.g., weather condition, season of the year, time of the day). Other information items are based on data collected by equipment which monitor the operation of the distribution system (e.g., number of phases affected, protective devices used, etc.). Once an outage is detected, crewmen are dispatched to restore/repair the system as well as to find the fault causes, if possible [3-6]. Duke Power has accumulated a huge database on outage information [7]. However, data is meaningless until they are interpreted correctly. There are many statistical tools that can be used to analyze this data. However, the statistics may lead to misleading results if interpreted carelessly because the actual problem may not be as simple as it appears to be.

In this paper, we will present an approach that uses statistical techniques to analyze and interpret the TOR data with respect to several factors such as time, weather condition, etc., and correlate the results with actual field experience in distribution systems. Outage data for the Durham Region in Duke Power System will be used in this paper for illustration purposes. Knowledge about distribution systems can help explain the statistical results, and the statistical results can provide quantitative information about the particular distribution system of interest. This type of quantitative information is very useful for improvement in customer service and different distribution system operations such as maintenance, construction and design, priority setting, and resource allocation. The approach taken in this paper can be easily modified for other utilities' TOR analysis. These analyses can provide a better understanding as to how the outage restoration time is affected by different influential factors. This knowledge can be very helpful for future improvements in customer services, distribution operations and planning.

II. Selected Factors and Statistical Tools for the Time of Outage Restoration Analysis

This paper focuses on several factors for TOR analysis and the factors are categorized into three main categories : (1) Time, (2) Consequence, and (3) External. Each category is further broken down into groups and classes for more detailed analysis. The general categories under investigation in this paper are schematically shown in Figure 1. (Note that there are other factors, such as fault location effects on ETOR, can be analyzed using similar approaches proposed in this paper).

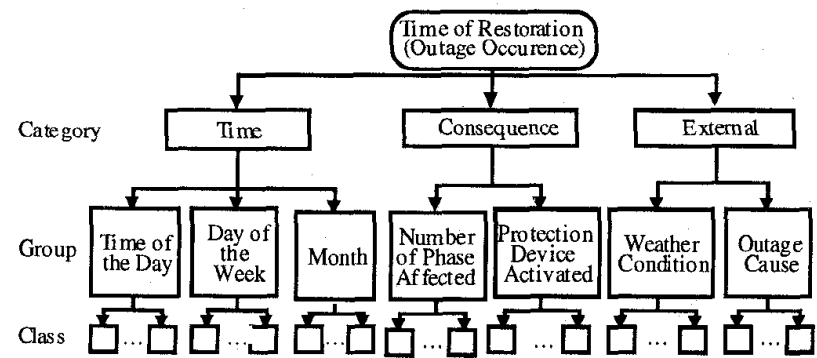

Fig. 1. The categories under investigation for Time of Restoration in this paper.
Many statistical tools are available for data analyses. We use graphical displays of TOR data to facilitate our discussion because "one picture is worth a thousand words". In order to illustrate the statistical measures used in this paper, Figure. 2 shows a typical histogram of TOR, with the quantile box drawn above the histogram. The median is shown as a line across the middle of the box, and the 25th and 75th quantiles are at the ends. The quantile boxes provide a summary of the distribution's shape. A mean diamond is also shown as a schematic that illustrates a sample mean and a $95 \%$ confidence interval. The line across each diamond represents the group mean. The width of each diamond represents the $95 \%$ confidence interval for each group. The diamond is very useful to examine whether the mean for each class under comparison is statistically different.

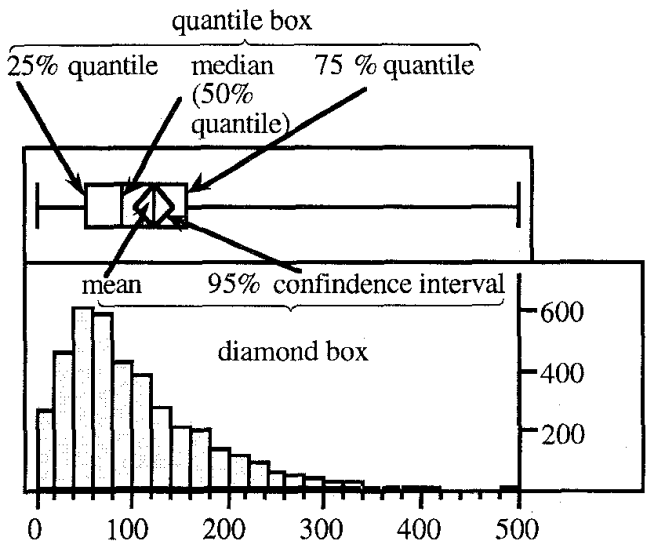

Fig. 2. Typical histogram plot of TOR.

The histogram in Figure 2 suggests that TOR is not normally distributed, as confirmed by a test for normality. The Kolmogorov-Smirnov statistic used for the normality test has a value of $\mathrm{D}=0.128$ [8], and the corresponding $p$ value is less than 0.01 . (Note that $p$-value is the probability of obtaining a more extreme value of $\mathrm{D}$ based on factors such as the $\chi^{2}$ value and degree of freedom (DF) of the problem.) The $p$-value obtained here indicates that TOR does not follow a normal distribution. We thus cannot use the conventional ANOVA technique to test for the equality of the mean or median of the TOR at different classes for the selected factors due to the violation of the normality assumption. In this paper, we use the Nonparametric One Way Analysis of Variance technique to analyze the TOR with respect to each of the selected factors. This procedure performs analysis of variance on ranks, is distribution free, and the assumption of normality is not made. In particular, the Kruskal-Wallis test is conducted here to test whether the Group medians are the same across Groups of the selected factors. For a more detailed description of Kruskal-Wallis method, please refer to [8].

The $\chi^{2}$ approximation to Kruskal-Wallis test, together with its $p$-value, will be given in the following sections when we discuss the inferences about TOR for each specific factor. A small $p$-value for the Kruskal-Wallis test indicates that the responses are different across the Group for the factor. On the other hand, a large $p$-value indicates that the responses are the same across the Group for the factor. The usual cutoff value for $p$-values is either 0.05 or 0.1 . The results of the $p$ values for our data for each factor are clearly much larger or 
smaller than the usual cutoff, thus the $p$-values provide a clear indication for our conclusions.

Besides determining the influential factors of TOR based on the $p$-values of the Kruskal-Wallis test, we also rank the importance of each factor for the TOR by $R^{2}$ measure, which ranges between 0 and $1 . R^{2}$ measures the proportionate reduction of total variation in the response variable associated with the use of independent variables, which represent each specific cause we consider in this paper. Higher $R^{2}$ indicates that the cause is more influential.

III. Non-Parametric One-Way Variance Analysis of the Time of Outage Restoration

In order to illustrate the approach of using statistical analyses for power distribution's outage restoration time analysis, the data set containing 4,323 outage records collected from Durham, North Carolina between 1992 and 1993 is used in this paper. The same methodology can be easily modified and applied to different distribution outage data. Note that the maximum TOR shown is 500 minutes ( 8 hours and 20 minutes), because outages which take more than 500 minutes are treated as abnormal situations (outliers in statistical terms) in this analysis and are disregarded in this TOR analysis.

\section{A. Time Factors}

The effect of the time of outage occurrence is first investigated. For a more detailed investigation, we further break down the time factor into three qualitative descriptions (groups) : (1) time of the day, (2) day of the week, and (3) month.

\section{TOR by Time of the Day}

In this study, the time of the day was divided into twelve time zones (classes) :

(1) midnight - 2am, (2) $2 \mathrm{am}$ - 4am, (3) 4am - 6am, (4) 6am 8am, (5) 8am - 10am, (6) 10am - noon, (7) noon - 2pm, (8) $2 \mathrm{pm}$ - 4pm, (9) 4pm - 6pm, (10) 6pm - 8pm, (11) 8pm $10 \mathrm{pm}$, (12) $10 \mathrm{pm}$ - midnight. The TOR distribution with respect to the twelve classes is graphically depicted in Figure 3. The plot is similar to the histogram shown in Figure 2 except that the count of the TOR observation is now shown as the density of the dot in the figure. In addition to the graphical display of the data, the means and variances are graphically displayed by the mean diamond boxes, as described in the previous paragraph. To test whether the median for TOR are the same for all different time zones, the Kruskal-Wallis test is performed. Its $\chi^{2}$ approximation has a value of 302.7535 with $\mathrm{DF}=11$ and the $p$-value $=0.0000$, which indicates that time of the day is statistically significant to the TOR.

Figure 3 clearly indicates that time of the day is an important factor for the TOR estimate. The statistical results agree with our intuition. For example, during normal working hours, there are more crewmen on duty and they are stationed either in the operation centers or in the field. They can respond faster to outage calls (outages usually have the first priority in distribution operations). During off-hour, there are less crewmen on duty and they are stationed in their homes on-call, so the response time will certainly be longer than during normal working hours. In summary, the TOR is inversely proportional to the availability and readiness of crewmen.

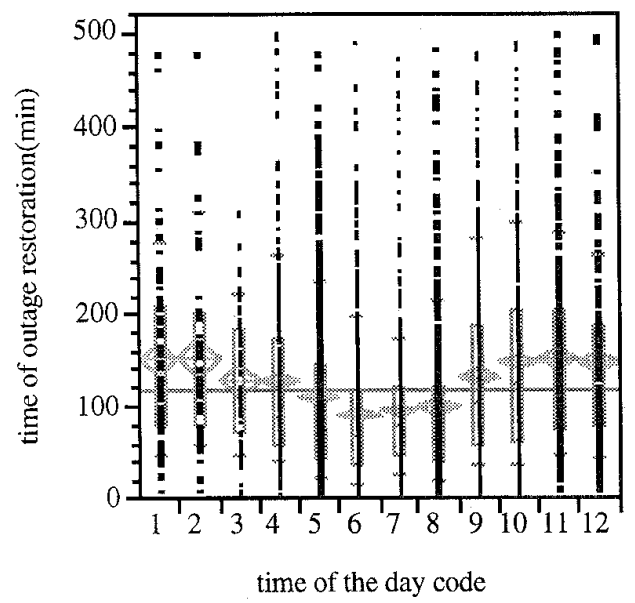

Fig. 3. Time of Outage Restoration by Time of the Day.

\section{TOR by Day of the Week}

The Time category was then grouped into Day of the Week : (0) Sunday, (1) Monday, (2) Tuesday, (3) Wednesday, (4) Thursday, (5) Friday, and (6) Saturday. The data distribution of the outage restoration time with respect to different day of the week is graphically depicted in Figure 4. The $\chi^{2}$ value is 6.6201 with $\mathrm{DF}=6$ and the $p$-value $=$ 0.3574 , which indicates that the day of the week is not statistical significant to TOR.

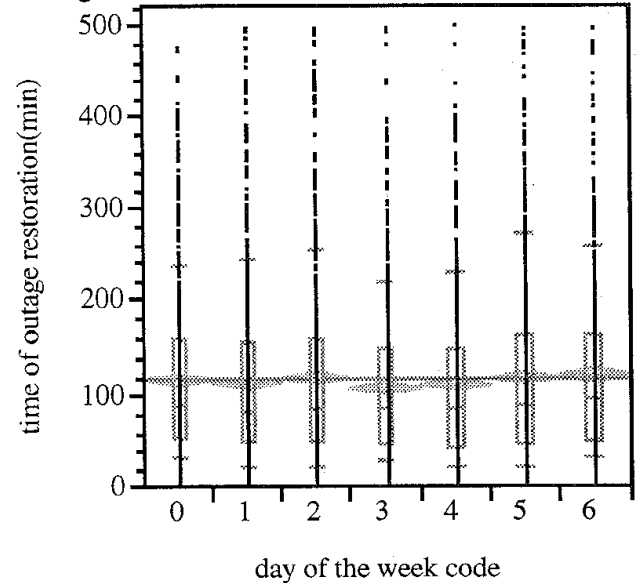

Fig. 4. Time of Outage Restoration by Day of the Week.

Figure 4 indicates that the mean values are very closed to each other (by inspection of the diamond box mean values and confidence levels of the TOR for different classes, as described in the previous section). The $\chi^{2}$ statistics also confirmed that the mean difference among different days of the week is not significant for the TOR estimate from a statistical point of view. These results contradict our intuition. One would think that the TOR should take longer time during the weekends than during weekdays because of the availability and the readiness of crew members - similar arguments used in the Time of the Day analysis. At the first sight, we thought this pattern occurred only for Durham's data. However, the investigation of other districts' data also showed the same statistical conclusions that the Day of the Week does not contribute significantly to the TOR estimate. This is an interesting phenomenon which we are currently investigating, 
in order to explain the discrepancy between the statistical results and our intuition.

\section{TOR by Month}

The Time category is also grouped into Months : (1) January, (2) February, (3) March, (4) April, (5) May, (6) June, (7) July, (8) August, (9) September, (10) October, (11) November, and (12) December. The TOR with respect to Month is graphically depicted in Figure 5. The $\chi^{2}$ value is 42.5849 with $\mathrm{DF}=11$ and the $p$-value $=0.0000$, which indicates that the month of the outage occurrence is influential to TOR.

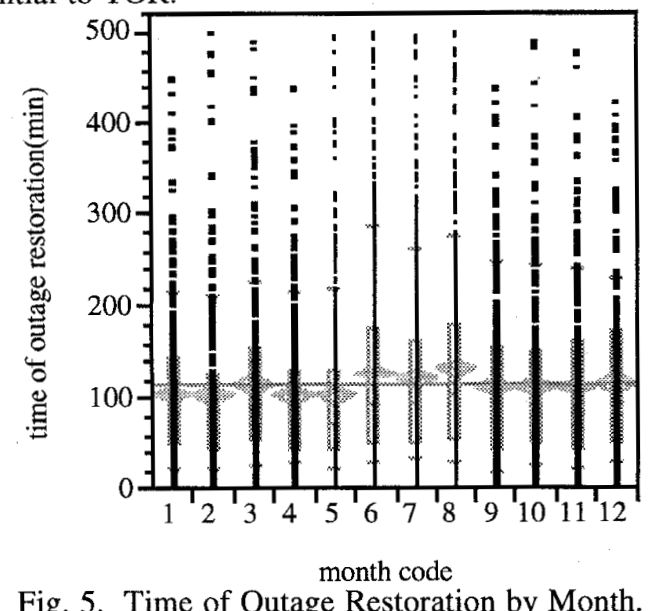

Fig. 5. Time of Outage Restoration by Month.

Figure 5 shows that there are variations between the TOR with respect to Month. In general, it takes a longer time to restore outages in the summer time (June - August) than in the winter time. The results agree with the reality in Duke Power Systems (North Carolina and South Carolina regions). In the summer, we have more severe weather conditions such as thunderstorms, which can cause a series of outages over a large region, than in the winter time. We do not have too many severe snow storms in the winter, which could have caused similar effects as the thunderstorms. The massive outages caused by severe weather conditions will take much longer to restore than a few isolated outages, because only a limited amount of manpower is available at a time. In addition to the individual outage restoration time, there are waiting periods for some outages to be restored. The authors believe that the utilities in the Northern region of the country will have longer TOR in the winters than in the summers because they may have more snow storms in the winter than thunderstorms in the summer. In summary, TOR is proportional to the weather severity.

\section{B. Consequence Factors}

\section{TOR by Number of Phase Affected}

Number of Phases Affected is one of the consequences of outage occurrences. The number of phases affected is coded as : (0) single-customer, (1) single-phase, (2) two-phases, and (3) three-phases. The single-customer can be on a singlephase, two-phase, or three-phase line. The outage is classified as a single-customer outage as long as the outage occurs on a service line rather than on the primary line. In this paper, we show the TOR for two regions, Durham and Chapel Hill, to demonstrate the local properties of different regions. Durham, which has approximately 90,000 customers, is a much larger region than Chapel Hill, which has approximately 35,000 customers. The TOR with respect to the number of phases affected in Durham is graphically shown in Figure 6a. The $\chi^{2}$ value of the number of phases affected with respect to TOR in Durham region is 89.3097 with DF 3 and the $p$-value is 0.0000 . The TOR with respect to the number of phase affected in Chapel Hill region is graphically shown in Figure $6 \mathrm{~b}$. The $\chi^{2}$ value of time of the day with respect to TOR in Chapel Hill region is 88.7600 with DF $=3$ and the $p$-value $=0.0000$, which indicates that the number of phases affected is also influential for the TOR.

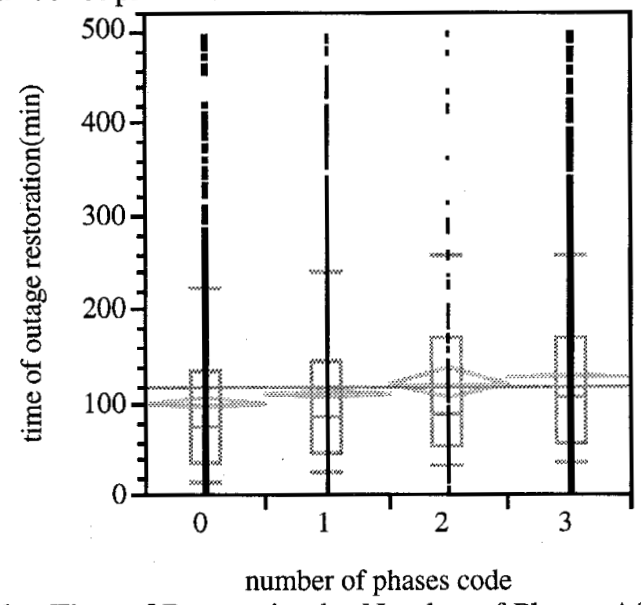

Fig. 6a. Time of Restoration by Number of Phases Affected

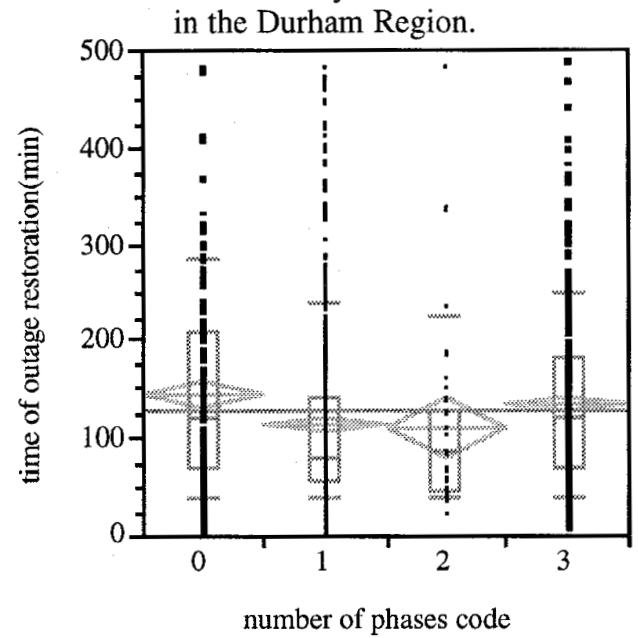

Fig. 6b. Time of Restoration by Number of Phases Affected in the Chapel Hill Region.

The number of phases in the circuits in general is proportional to the service area they cover (except for the single customer circuit, which we will explain later). A three-phase line is normally larger than a two-phase line, which is larger than a single-phase line. Thus, it takes much more time to locate a three-phase circuit outage than a twophase outage than a single-phase outage, as indicated in Figure 6a for Durham. However, in the Chapel Hill region, Figure $6 b$ shows that the TOR for two-phase circuit outages is shorter than single-phase circuit outages. Actually, there are much less two-phase circuits than single-phase circuits in the Duke Power system, which significantly reduces the search time for two-phase outages. Furthermore, there are not 
too many two-phase outages in Chapel Hill, thus the statistical estimate of the TOR is not so representative, as indicated by the wide diamond box confidence interval.

In addition, Figure $6 b$ indicates that single-customer outage takes longer time to restore than other types of outages, which is a little surprising. After further investigation, the statistical results actually agree with the actual situations. The TOR can be thought of as both the time required to find the outage location and the time to fix the outage. For single-customer outages, the outage locations can be pin-pointed quickly, thus taking much less time to find the outage location. However, in the Chapel Hill region, many of the single-customer services are underground, while the single-phase, two-phase, and threephase lines are in general overhead. By and large, it takes much longer time to fix an underground outage than an overhead outage. The TOR for single-customer outages is mainly spent on fixing the outages instead of finding them.

In summary, the TOR is proportional to the size of the area covered by the circuits in terms of search time, and also to the type of distribution lines, whether underground or overhead, in terms of fixing time.

\section{TOR by Activated Protection Devices}

An outage will activate protection devices such as blow fuses and circuit breakers. This section discusses the TOR with respect to different protection devices which are activated by the outages. The protection devices are coded as : (0) Transmission Device, (1) Station Circuit Breaker, (2) Line Reclosure, (3) Primary Fuse, (4) Transformer Fuse, (5) Transformer CSP/Other, (6) Panel/Meter Base, (7) SEC/SVC Self Clearing, and (8) Manual Device. The TOR with respect to these protection device codes is graphically depicted in Figure 7. The $\chi^{2}$ value is 359.6365 with DF $=8$ and the $p$ value $=0.0000$, which indicates that activated protection devices provide statistically significant information for TOR.

Figure 7 clearly indicates that TOR is a function of the activated protection devices. However, the TOR is not only a function of how easy it is to restore the protection devices. More importantly, TOR is a function of how significant the protection device is to the system. Suppose a thunderstorm caused many protection devices to activate, including Static Circuit Breakers (code 1) and primary fuses (code 3). Physically, it may take less time to restore a primary fuse than a Static Circuit Breaker (such as OCB). However, from the distribution system reliability viewpoint, we want to restore power to most of our customers as soon as possible. Thus we need to set priorities for protection device restorations. The activation of an OCB will affect an entire circuit, say 5,000 customers, while a primary fuse may affect only a section of a feeder, say 10 customers. Therefore, the OCB protection device has a much higher priority than the primary fuses, thus the statistics showed that OCB takes less time to restore than other protection devices. Other than the priority issue of restoration, the actual time required to restore the protection device will also come into the picture. For example, SEC/SVC Self Clearing protection devices (code 7) are usually buried underground, which takes much longer to repair than a overhead fuse failure. In summary, the TOR is proportional to the importance of the protection device in terms of the system reliability as the first concern; then other factors such as to the actual time required to re-install the protection devices will be considered.

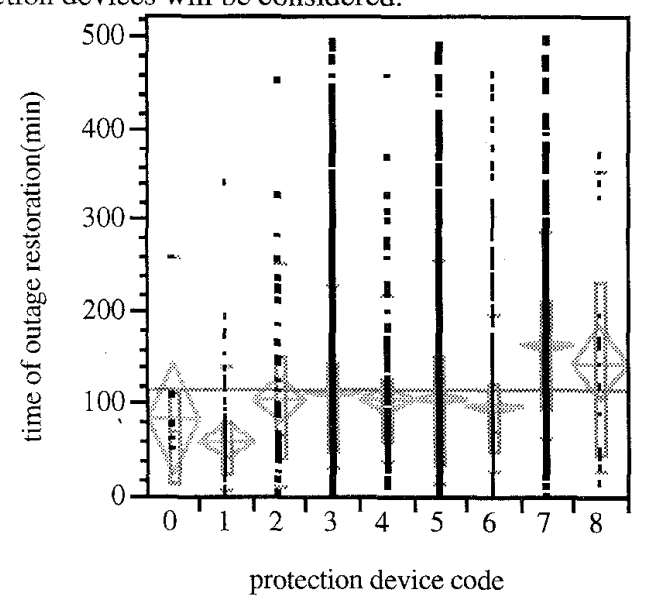

Fig. 7. Time of Outage Restoration by Protection Device Code.

\section{External Factors}

External factors such as weather conditions, outage causes, etc. can affect the outage restoration time. This section discusses the TOR with respect to weather conditions and outage causes.

\section{TOR by Weather Conditions}

In this study, the weather conditions are categorized into : (0) Fair, (1) Cold, (2) Rain, (3) Wind, (4) Wind \& Lightning, (5) Lightning, (7) Snow, (8) Ice, (9) Hot, and (10) Fog.

The TOR with respect to weather conditions is graphically depicted in Figure 8. The $\chi^{2}$ value is 125.5354 with $\mathrm{DF}=9$ and the $p$-value $=0.0000$, which indicates that the weather condition provides useful information for TOR.

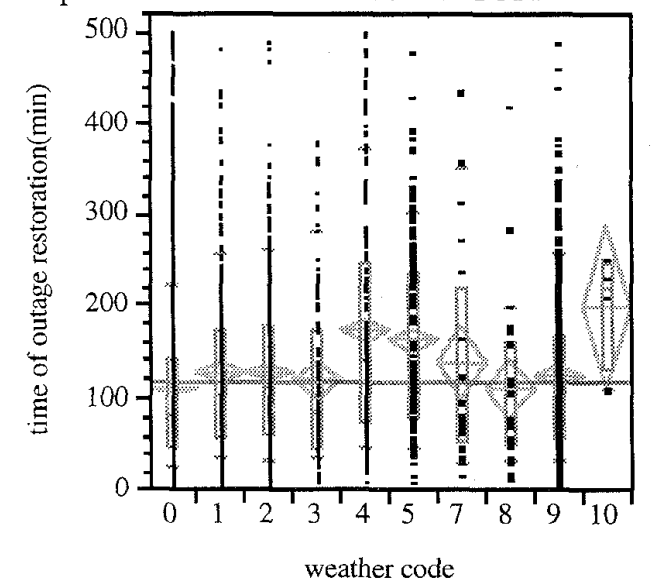

Fig. 8. Time of Outage Restoration by Weather Code.

Figure 8 indicates that the weather condition is a strongly influential factor for the TOR. Under bad weather conditions, e.g. stormy weather with high winds, lightning, etc., the outage restoration time takes much longer than in fair weather conditions. There are many reasons that explain why the weather condition can significantly affect the outage restoration time. To name a few : (1) It takes longer for crewman to restore an outage in bad weather than in fair weather; (2) stormy weather may cause many different outages 
in a short period of time and there is a long waiting period before the crewman can start restoring some of the outages. The mean values of TOR of snow (class 7) and ice (class 8) conditions in Fig. 8 indicate that snow causes a longer outage restoration time than ice. Intuitively, ice conditions is in general cause longer TOR than snow conditions due to reasons such as slippery roads. However, the data under analyses is in the Duke Power territory, which is located in the southern part of United States. We do not experience too much snow and ice conditions. The width of the diamond boxes, which indicates the variance of the mean estimate, for snow and ice conditions in Fig. 8 is very wide and has a large overlap on TOR for each others. This indicates that we cannot draw statistical conclusions of TOR in terms of the mean estimate to compare the snow and ice conditions. In summary, TOR is proportional to the severity of weather conditions - similar arguments as for month factor.

\section{Types of Outage Causes}

This section analyze the TOR with respect to different outage causes. The causes under consideration are coded as : (0) Customer Problem, (1) DPCO Accident, (2) Public Accidents, (3) Danger Trees, (4) Trees, Vines, (5) Animals, (6) Overload, (7) Lightning Strikes, BIL, (8) UG Services, (9) UG Primary, (10) Transformer Failure, (11) HPP/MHP Breaker, (12) Meter Socket/HPP/MHP, (13) Bad Secondary Connector, (14) Improper installation, (15) Natural Deterioration, (16) Vandalism, and (17) Other code. The TOR with respect to the cause codes is graphically depicted in Figure 9. The $\chi^{2}$ value is 1087.9372 with $\mathrm{DF}=17$ and $p$ value $=0.0000$, which indicates that outage causes are influential to TOR.

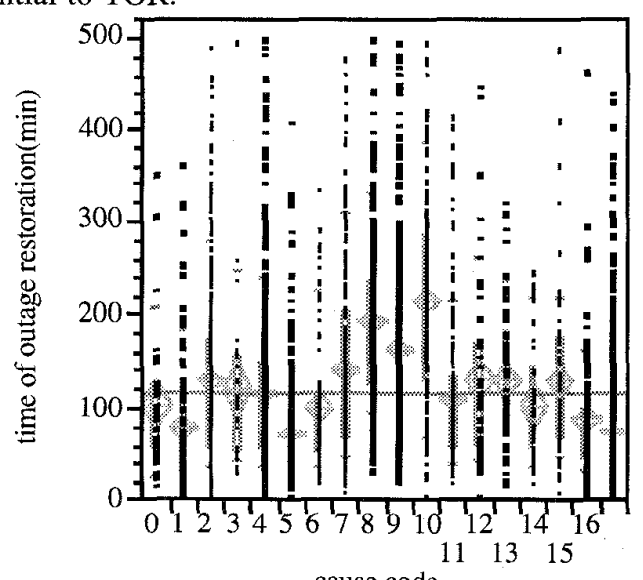

Fig. 9. Time of Outage Restoration by Cause Code.

As indicated in Figure 9, the cause of an outage correlates strongly with the TOR. Actually, the outage cause is the most correlated factor among all the factors discussed previously. We will discuss the reason behind this strong correlation in the next section, where we discuss about the ranking of the factors' influences for estimating TOR.

D. $R^{2}$ Analysis

Among all the influential factors under investigation, most of them are found statistically significant for the TOR. In this section, we use the $R^{2}$ value as described in Section II to evaluate and rank the degree of influence of the selected factors. The $R^{2}$ values with respect to the different factors for TOR estimate are listed in Table 1 in descending order.

As indicated previously, outage causes is the most influential factor for TOR. In [3], the authors have presented their work on estimating the outage causes based on the other factors considered in this paper, achieving satisfactory results. This implies that the factors under consideration may be correlated with each other as well. For example, an animal outage usually activate a single-phase primary fuse in a nice morning [9]. The crewman can drive to the fault location, change the fuse and restore the outage efficiently. However, if a distribution transformer fails, the crewman needs to take down the transformer, and install a new transformer. Apparently, this process takes more time and effort to restore the outage.

It is not surprising that the Day of the Week category has the lowest $R^{2}$ value because the classes under these category do not really provide information for the TOR, as indicated in the $\chi^{2}$ statistics discussed previously.

Table $1 . R^{2}$ values of chosen factors with respect to the TOR.

\begin{tabular}{|c|c|}
\hline Factors & $\boldsymbol{R}^{\mathbf{2}}$ \\
\hline Outage Cause & 0.2130 \\
\hline Time of the Day & 0.0567 \\
\hline Protection Device Activated & 0.0550 \\
\hline Weather Conditions & 0.0295 \\
\hline Number of Phase Affected & 0.0137 \\
\hline Month & 0.0110 \\
\hline Day of the Week & 0.0023 \\
\hline
\end{tabular}

\section{Conclusion}

In this paper, we have analyzed the time of outage restoration with respect to several selected factors. Both statistical, quantitative results and field engineering insights have been discussed. We used the quantitative results to support our field experience insights to this problem (which is qualitative information); likewise, we used our field experience to interpret the statistical results so that the statistical results will not be mis-interpreted due to the lack of field experience. The methodology and general discussion in the paper will be useful for research and development on the Time of Outage Restoration issues in distribution systems, such as modeling development for customer service improvement.

\section{Acknowledgment}

The authors of this paper would like to acknowledge the support of Electric Power Research Center project grant 531326, North Carolina State University and Duke Power Company project grant 5-30585.

\section{Reference}

[1] M.-y. Chow and L. S. Taylor, "Application of Information Technology to Distribution System Operations," Proceedings of Electric Power Systems Infrastructure Symposium, Pullman, WA, 1994.

[2] H. M. Rustebakke, Electric Utility Systems and Pactices, 4 ed. New York: A Wiley-Interscience Publication, 1983.

[3] M.-y. Chow, S. O. Yee, and L. S. Taylor, "Recognizing Animal-Caused Faults in Power Distribution Systems Using 
Artificial Neural Networks," IEEE Transactions on Power Delivery, vol. 8, pp. 1268-1274, 1993.

[4] R. Balakrishnan and A. Pahwa, "A Computer Assisted Intelligent Storm Outage Evaluation for Power Distribution Systems," IEEE Transactions on Power Delivery, vol. 5, pp. $1591-1597,1990$.

[5] K. Tomsovic, P. Ackerman, and S. Pope, "An Expert System as a Dispatchers' Aid for Isolation of Line Section Faults," IEEE Transactions on Power Delivery, vol. 2, pp. $736-743,1987$.

[6] C. Liu, S. J. Lee, and S. S. Venkata, "An Expert System Opeartional Aid for Restoration and Loss Reduction of Distribution Systems," presented at IEEE 1987 PICA Conference, 1987.

[7] M.-y. Chow and L. S. Taylor, "A Novel Approach for Distribution Fault Analysis," IEEE Transactions on Power Delivery, vol. 8, pp. 1882-1889, 1993.

[8] E. R. Dougherty, Probability and Statistics for the Engineering, Computing, and Physical Sciences: Prentice Hall, 1990.

[9] M.-y. Chow and L. S. Taylor, "On the Distribution System Animal-Caused Faults," IEEE Transaction on Power Delivery, vol. 10, 1995.

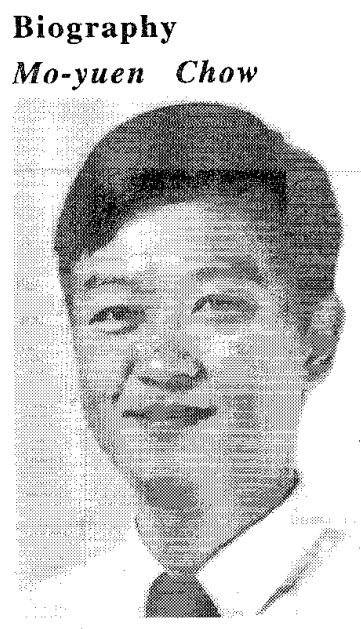

Mo-yuen Chow earned his B.S. degree at the University of Wisconsin-Madison (1982); $M$. Eng. degree (1983) and Ph.D. degrees at Cornell University (1987), all in Electrical Engineering. Upon completion of his Ph.D., Dr. Chow joined the faculty of North Carolina State University in Raleigh, $\mathrm{NC}$, where he is presently an Associate Professor in the Department of Electrical and Computer Engineering. Dr. Chow has also worked as a consultant for Taiwan Power Company, J.W. Harley Company, and as a faculty intern at Duke Power Company.

Since 1987, Dr. Chow has been working as a Principal Investigator in several projects in the areas of system monitoring, fault detection and control, applications of artificial neural network and fuzzy logic to power engineering. Dr. Chow served as a guest editor of IEEE Transactions on Industrial Electronics Special Issue on Application of Intelligent Systems to Industrial Electronics. He is currently an Associate Editor of the IEEE Transactions on Industrial Electronics. He is also listed in Who's Who in Asian Americans.

\section{Leroy S. Taylor}

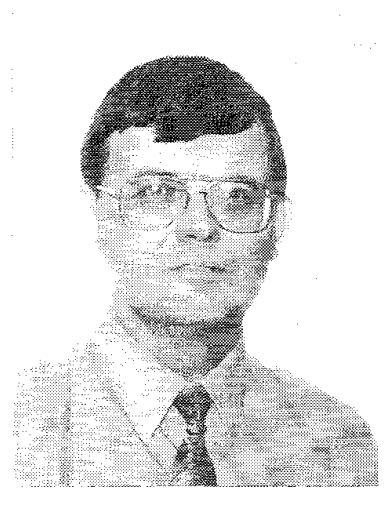

Leroy S. Taylor is a Senior Distribution Engineer for Duke Power Company, and is a registered professional engineer in North Carolina. He was born in 1949 in Greenville, N.C. and received a B.A. degree (Physics) from the University of North Carolina in 1971. Joining Duke Power in 1977, he acquired extensive experience in distribution system engineering, operation, and construction. Since 1987 he has conducted intensive investigations on the cause of power quality disturbances which originate in the distribution system. He has also redesigned several Duke Power mainframe reporting systems used to evaluate and improve distribution system reliability and power quality.

\section{Mo-suk Chow}

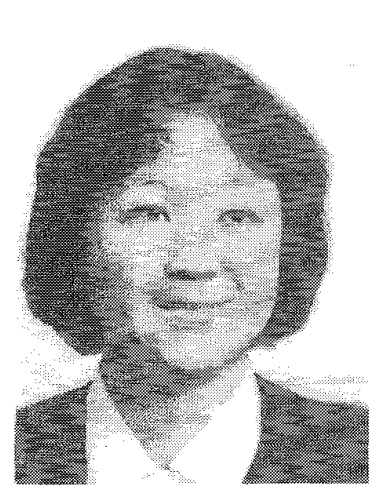

Mosuk Chow got her Ph.D. degree in the field of Statistics from Cornell University 1983. During her graduate study, her expertise was in the area of decision theory and statistical computing. Since then her research interest branched out and emcompassed a diverse applications of statistical method to various problems such as efficient estimation of superimposed exponential signals, capture and recapture problems and regression problems. Dr. Chow had taught at Northeastern University and Penn State University. She Currently works for C M Soft Company as a senior research associate and is an adjunct faculty of the Department of Management Science and Information System of the Penn State University. 